\title{
Existence results for nonlocal boundary value problems of fractional differential equations and inclusions with strip conditions
}

\author{
Bashir Ahmad $^{1 *}$ and Sotiris K Ntouyas ${ }^{2}$
}

\author{
* Correspondence: \\ bashir_qau@yahoo.com \\ 'Department of Mathematics, \\ Faculty of Science, King Abdulaziz \\ University, P.O. Box 80203, Jeddah \\ 21589, Saudi Arabia \\ Full list of author information is \\ available at the end of the article
}

\begin{abstract}
This article studies a new class of nonlocal boundary value problems of nonlinear differential equations and inclusions of fractional order with strip conditions. We extend the idea of four-point nonlocal boundary conditions

$(x(0)=\sigma x(\mu), x(1)=\eta x(v), \sigma, \eta \in \mathbb{R}, 0<\mu, v<1)$ to nonlocal strip conditions

of the form: $x(0)=\sigma \int_{\alpha}^{\beta} x(s) d s, x(1)=\eta \int_{\gamma}^{\delta} x(s) d s, 0<\alpha<\beta<\gamma<\delta<1$.

These strip conditions may be regarded as six-point boundary conditions. Some new existence and uniqueness results are obtained for this class of nonlocal problems by using standard fixed point theorems and Leray-Schauder degree theory. Some illustrative examples are also discussed.
\end{abstract}

MSC 2000: 26A33; 34A12; 34A40.

Keywords: fractional differential equations, fractional differential inclusions, nonlocal boundary conditions, fixed point theorems, Leray-Schauder degree

\section{Introduction}

The subject of fractional calculus has recently evolved as an interesting and popular field of research. A variety of results on initial and boundary value problems of fractional order can easily be found in the recent literature on the topic. These results involve the theoretical development as well as the methods of solution for the fractional-order problems. It is mainly due to the extensive application of fractional calculus in the mathematical modeling of physical, engineering, and biological phenomena. For some recent results on the topic, see [1-19] and the references therein.

In this article, we discuss the existence and uniqueness of solutions for a boundary value problem of nonlinear fractional differential equations and inclusions of order $q \in$ $(1,2]$ with nonlocal strip conditions. As a first problem, we consider the following boundary value problem of fractional differential equations

$$
\left\{\begin{array}{c}
{ }^{c} D^{q} x(t)=f(t, x(t), \quad 0<t<1, \quad 1<q \leq 2, \\
x(0)=\sigma \int_{\alpha}^{\beta} x(s) d s, \quad x(1)=\eta \int_{\gamma}^{\delta} x(s) d s, \quad 0<\alpha<\beta<\gamma<\delta<1,
\end{array}\right.
$$

\section{Springer}

(c) 2012 Ahmad and Ntouyas; licensee Springer. This is an Open Access article distributed under the terms of the Creative Commons Attribution License (http://creativecommons.org/licenses/by/2.0), which permits unrestricted use, distribution, and reproduction in any medium, provided the original work is properly cited. 
where ${ }^{c} D^{q}$ denotes the Caputo fractional derivative of order $q$, $f:[0,1] \times \mathbb{R} \rightarrow \mathbb{R}$ is a given continuous function and $\sigma, \eta$ are appropriately chosen real numbers.

The boundary conditions in the problem (1.1) can be regarded as six-point nonlocal boundary conditions, which reduces to the typical integral boundary conditions in the limit $\alpha, \gamma \rightarrow 0, \beta, \delta \rightarrow 1$. Integral boundary conditions have various applications in applied fields such as blood flow problems, chemical engineering, thermoelasticity, underground water flow, population dynamics, etc. For a detailed description of the integral boundary conditions, we refer the reader to the articles [20,21] and references therein. Regarding the application of the strip conditions of fixed size, we know that such conditions appear in the mathematical modeling of real world problems, for example, see $[22,23]$.

As a second problem, we study a two-strip boundary value problem of fractional differential inclusions given by

$$
\left\{\begin{array}{c}
{ }^{c} D^{q} x(t) \in F(t, x(t)), \quad 0<t<1, \quad 1<q \leq 2, \\
x(0)=\sigma \int_{\alpha}^{\beta} x(s) d s, \quad x(1)=\eta \int_{\gamma}^{\delta} x(s) d s, \quad 0<\alpha<\beta<\gamma<\delta<1,
\end{array}\right.
$$

where $F:[0,1] \times \mathbb{R} \rightarrow \mathcal{P}(\mathbb{R})$ is a multivalued map, $\mathcal{P}(\mathbb{R})$ is the family of all subsets of $\mathbb{R}$.

We establish existence results for the problem (1.2), when the right-hand side is convex as well as non-convex valued. The first result relies on the nonlinear alternative of Leray-Schauder type. In the second result, we shall combine the nonlinear alternative of Leray-Schauder type for single-valued maps with a selection theorem due to Bressan and Colombo for lower semicontinuous multivalued maps with nonempty closed and decomposable values, while in the third result, we shall use the fixed point theorem for contraction multivalued maps due to Covitz and Nadler.

The methods used are standard, however their exposition in the framework of problems (1.1) and (1.2) is new.

\section{Linear problem}

Let us recall some basic definitions of fractional calculus [24-26].

Definition 2.1 For at least n-times continuously differentiable function $g:[0, \infty) \rightarrow \mathbb{R}$, the Caputo derivative of fractional order $q$ is defined as

$$
{ }^{c} D^{q} g(t)=\frac{1}{\Gamma(n-q)} \int_{0}^{t}(t-s)^{n-q-1} g^{(n)}(s) d s, \quad n-1<q<n, n=[q]+1,
$$

where $[q]$ denotes the integer part of the real number $q$.

Definition 2.2 The Riemann-Liouville fractional integral of order $q$ is defined as

$$
I^{q} g(t)=\frac{1}{\Gamma(q)} \int_{0}^{t} \frac{g(s)}{(t-s)^{1-q}} d s, \quad q>0,
$$

provided the integral exists.

By a solution of (1.1), we mean a continuous function $x(t)$ which satisfies the equation ${ }^{c} D^{q} x(t)=f(t, x(t)), 0<t<1$, together with the boundary conditions of (1.1). 
To define a fixed point problem associated with (1.1), we need the following lemma, which deals with the linear variant of problem (1.1).

Lemma 2.3 For a given $g \in C([0,1], \mathbb{R})$, the solution of the fractional differential equation

$$
{ }^{c} D^{q} x(t)=g(t), \quad 1<q \leq 2
$$

subject to the boundary conditions in (1.1) is given by

$$
\begin{aligned}
& x(t)=\frac{1}{\Gamma(q)} \int_{0}^{t}(t-s)^{q-1} g(s) d s \\
& +\frac{\sigma}{\Delta}\left[-\left(\frac{\eta}{2}\left(\delta^{2}-\gamma^{2}\right)-1\right)+t(\eta(\delta-\gamma)-1)\right] \int_{\alpha}^{\beta}\left(\int_{0}^{s} \frac{(s-m)^{q-1}}{\Gamma(q)} g(m) d m\right) d s \\
& +\frac{1}{\Delta}\left[\frac{\sigma}{2}\left(\beta^{2}-\alpha^{2}\right)-(\sigma(\beta-\alpha)-1) t\right]\left\{\eta \int_{\gamma}^{\delta}\left(\int_{0}^{s} \frac{(s-m)^{q-1}}{\Gamma(q)} g(m) d m\right) d s\right. \\
& \left.-\int_{0}^{1} \frac{(1-s)^{q-1}}{\Gamma(q)} g(s) d s\right\},
\end{aligned}
$$

where

$$
\Delta=\left[\frac{\eta}{2}\left(\delta^{2}-\gamma^{2}\right)-1\right][\sigma(\beta-\alpha)-1]-\left[\frac{\sigma}{2}\left(\beta^{2}-\alpha^{2}\right)\right][\eta(\delta-\gamma)-1] \neq 0 .
$$

Proof. It is well known that the solution of (2.1) can be written as [24]

$$
x(t)=I^{q} g(t)-c_{0}-c_{1} t=\int_{0}^{t} \frac{(t-s)^{q-1}}{\Gamma(q)} g(s) d s-c_{0}-c_{1} t .
$$

where $c_{0}, c_{1} \in \mathbb{R}$ are constants. Applying the boundary conditions given in (1.1), we find that

$$
\begin{aligned}
(\sigma(\beta-\alpha)-1) c_{0}+\frac{\sigma}{2}\left(\beta^{2}-\alpha^{2}\right) c_{1}= & \sigma \int_{\alpha}^{\beta}\left(\int_{0}^{s} \frac{(s-m)^{q-1}}{\Gamma(q)} g(m) d m\right) d s, \\
(\eta(\delta-\gamma)-1) c_{0}+\left(\frac{\eta}{2}\left(\delta^{2}-\gamma^{2}\right)-1\right) c_{1}= & \eta \int_{\gamma}^{\delta}\left(\int_{0}^{s} \frac{(s-m)^{q-1}}{\Gamma(q)} g(m) d m\right) d s \\
& -\int_{0}^{1} \frac{(1-s)^{q-1}}{\Gamma(q)} g(s) d s .
\end{aligned}
$$

Solving these equations simultaneously, we find that

$$
\begin{aligned}
c_{0}= & \frac{1}{\Delta}\left[\left(\frac{\eta}{2}\left(\delta^{2}-\gamma^{2}\right)-1\right) \sigma \int_{\alpha}^{\beta}\left(\int_{0}^{s} \frac{(s-m)^{q-1}}{\Gamma(q)} g(m) d m\right) d s\right. \\
& \left.-\frac{\sigma}{2}\left(\beta^{2}-\alpha^{2}\right)\left\{\eta \int_{\gamma}^{\delta}\left(\int_{0}^{s} \frac{(s-m)^{q-1}}{\Gamma(q)} g(m) d m\right) d s-\int_{0}^{1} \frac{(1-s)^{q-1}}{\Gamma(q)} g(s) d s\right\}\right],
\end{aligned}
$$




$$
\begin{aligned}
c_{1}= & \frac{1}{\Delta}\left[-(\eta(\delta-\gamma)-1) \sigma \int_{\alpha}^{\beta}\left(\int_{0}^{s} \frac{(s-m)^{q-1}}{\Gamma(q)} g(m) d m\right) d s\right. \\
& \left.+(\sigma(\beta-\alpha)-1)\left\{\eta \int_{\gamma}^{\delta}\left(\int_{0}^{s} \frac{(s-m)^{q-1}}{\Gamma(q)} g(m) d m\right) d s-\int_{0}^{1} \frac{(1-s)^{q-1}}{\Gamma(q)} g(s) d s\right\}\right] .
\end{aligned}
$$

Substituting the values of $c_{0}$ and $c_{1}$ in (2.3), we obtain the solution (2.2). $\square$

\section{Existence results for single-valued case}

Let $\mathcal{C}=C([0,1], \mathbb{R})$ denotes the Banach space of all continuous functions from $[0,1]$ $\rightarrow \mathbb{R}$ endowed with the norm defined by $\|x\|=\sup \{|x(t)|, t \in[0,1]\}$.

In view of Lemma 2.3, we define an operator $\mathbf{F}: \mathcal{C} \rightarrow \mathcal{C}$ by

$$
\begin{aligned}
& (\mathbf{F} x)(t) \\
& =\frac{1}{\Gamma(q)} \int_{0}^{t}(t-s)^{q-1} f(s, x(s)) d s \\
& +\frac{\sigma}{\Delta \Gamma(q)}\left[-\left(\frac{\eta}{2}\left(\delta^{2}-\gamma^{2}\right)-1\right)+t(\eta(\delta-\gamma)-1)\right] \int_{\alpha}^{\beta}\left(\int_{0}^{s}(s-m)^{q-1} f(m, x(m)) d m\right) d s \\
& +\frac{\eta}{\Delta \Gamma(q)}\left[\frac{\sigma}{2}\left(\beta^{2}-\alpha^{2}\right)-(\sigma(\beta-\alpha)-1) t\right] \int_{\gamma}^{\delta}\left(\int_{0}^{s}(s-m)^{q-1} f(m, x(m)) d m\right) d s \\
& -\frac{1}{\Delta \Gamma(q)}\left[\frac{\sigma}{2}\left(\beta^{2}-\alpha^{2}\right)-(\sigma(\beta-\alpha)-1) t\right] \int_{0}^{1}(1-s)^{q-1} f(s, x(s)) d s .
\end{aligned}
$$

Observe that the problem (1.1) has solutions if and only if the operator equation $\mathbf{F} x$ $=x$ has fixed points.

For the forthcoming analysis, we need the following assumptions:

(A) $|f(t, x)-f(t, y)| \leq L|x-y|, \forall t \in[0,1], L>0, x, y \in \mathbb{R}$;

$\left(\mathbf{A}_{2}\right)|f(t, x)| \leq \mu(t), \forall(t, x) \in[0,1] \times \mathbb{R}$, and $\mu \in C\left([0,1], \mathbb{R}^{+}\right)$.

For convenience, let us set

$$
\Lambda=\frac{1}{\Gamma(q+1)}\left(1+\frac{\Delta_{2}|\sigma|\left(\beta^{q+1}-\alpha^{q+1}\right)+\Delta_{1}|\eta|\left(\delta^{q+1}-\gamma^{q+1}\right)+(q+1) \Delta_{1}}{(q+1)|\Delta|}\right),
$$

where

$$
\left|\frac{\sigma}{2}\left(\beta^{2}-\alpha^{2}\right)\right|+|(\sigma(\beta-\alpha)-1)|:=\Delta_{1},\left|\left(\frac{\eta}{2}\left(\delta^{2}-\gamma^{2}\right)-1\right)\right|+|(\eta(\delta-\gamma)-1)|:=\Delta_{2} .
$$

Theorem 3.1 Assume that $f:[0,1] \times \mathbb{R} \rightarrow \mathbb{R}$ is a jointly continuous function and satisfies the assumption $\left(A_{1}\right)$ with $L<1 / \Lambda$, where $\Lambda$ is given by (3.2). Then the boundary value problem (1.1) has a unique solution.

Proof. Setting $\sup _{t \in[0,1]}|f(t, 0)|=M<\infty$ and choosing $r \geq \frac{\Lambda M}{1-L \Lambda}$, we show that $\mathbf{F} B_{r} \subset B_{r}$, where $B_{r}=\{x \in \mathcal{C}:\|x\| \leq r\}$. For $x \in B_{r}$, we have 


$$
\begin{aligned}
& \|(\mathbf{F} x)\| \leq \sup _{t \in[0,1]}\left\{\frac{1}{\Gamma(q)} \int_{0}^{t}(t-s)^{q-1}|f(s, x(s))| d s\right. \\
& +\left|\frac{\sigma}{\Delta \Gamma(q)}\left[-\left(\frac{\eta}{2}\left(\delta^{2}-\gamma^{2}\right)-1\right)+t(\eta(\delta-\gamma)-1)\right]\right| \\
& \times \int_{\alpha}^{\beta}\left(\int_{0}^{s}(s-m)^{q-1}|f(m, x(m))| d m\right) d s \\
& +\left|\frac{\eta}{\Delta \Gamma(q)}\left[\frac{\sigma}{2}\left(\beta^{2}-\alpha^{2}\right)-(\sigma(\beta-\alpha)-1) t\right]\right| \int_{\gamma}^{\delta}\left(\int_{0}^{s}(s-m)^{q-1}|f(m, x(m))| d m\right) d s \\
& \left.+\left|\frac{1}{\Delta \Gamma(q)}\left[\frac{\sigma}{2}\left(\beta^{2}-\alpha^{2}\right)-(\sigma(\beta-\alpha)-1) t\right]\right| \int_{0}^{1}(1-s)^{q-1}|f(s, x(s))| d s\right\} \\
& \leq \sup _{t \in[0,1]}\left\{\frac{1}{\Gamma(q)} \int_{0}^{t}(t-s)^{q-1}(|f(s, x(s))-f(s, 0)|+|f(s, 0)|)|| d s\right. \\
& \left.+\frac{|\sigma|}{|\Delta| \Gamma(q)} \Delta_{2} \int_{\alpha}^{\beta}\left(\int_{0}^{s} s-m\right)^{q-1}(|f(m, x(m))-f(m, 0)|+|f(m, 0)|) d m\right) d s \\
& \left.+\frac{|\eta|}{|\Delta| \Gamma(q)} \Delta_{1} \int_{\gamma}^{\beta}\left(\int_{0}^{s} s-m\right)^{q-1}(|f(m, x(m))-f(m, 0)|+|f(m, 0)|) d m\right) d s \\
& \left.+\frac{1}{|\Delta| \Gamma(q)} \Delta_{1} \int_{0}^{1}(1-s)^{q-1}(|f(s, x(s))-f(s, 0)|+|f(s, 0)|) d s\right\} \\
& \leq(L r+M) \sup _{t \in[0,1]}\left\{\frac{1}{\Gamma(q)} \int_{0}^{t}(t-s)^{q-1} d s+\frac{|\sigma|}{|\Delta| \Gamma(q)} \Delta_{2} \int_{\alpha}^{\beta}\left(\int_{0}^{s}(s-m)^{q-1} d m\right) d s\right. \\
& \left.+\frac{|\eta|}{|\Delta| \Gamma(q)} \Delta_{1} \int_{\gamma}^{\delta}\left(\int_{0}^{s}(s-m)^{q-1} d m\right) d s+\frac{1}{|\Delta| \Gamma(q)} \Delta_{1} \int_{0}^{1}(1-s)^{q-1} d s\right\} \\
& \leq \frac{(L r+M)}{\Gamma(q+1)}\left(1+\frac{\Delta_{2}|\sigma|\left(\beta^{q+1}-\alpha^{q+1}\right)}{(q+1)|\Delta|}+\frac{\Delta_{1}|\eta|\left(\delta^{q+1}-\gamma^{q+1}\right)}{(q+1)|\Delta|}+\frac{\Delta_{1}}{|\Delta|}\right) \\
& =(L r+M) \Lambda \leq r \text {. }
\end{aligned}
$$

Now, for $x, y \in \mathcal{C}$ we obtain

$$
\begin{aligned}
& \quad\|(\mathbf{F} x)(t)-(\mathbf{F} y)\| \\
& \leq \sup _{t \in[0,1]}\left\{\frac{1}{\Gamma(q)} \int_{0}^{t}(t-s)^{q-1}|f(s, x(s))-f(s, y(s))| d s\right. \\
& +\frac{|\sigma|}{|\Delta| \Gamma(q)} \Delta_{2} \int_{\alpha}^{\beta}\left(\int_{0}^{s}(s-m)^{q-1}|f(m, x(m))-f(m, \gamma(m))| d m\right) d s \\
& \left.+\frac{|\eta|}{|\Delta| \Gamma(q)} \Delta_{1} \int_{\gamma}^{\delta}\left(\int_{0}^{s}(s-m)^{q-1}|f(m, x(m))-f(m, \gamma(m))| d m\right) d s\right\} \\
& +\frac{1}{|\Delta| \Gamma(q)} \Delta_{1} \int_{0}^{1}(1-s)^{q-1}|f(s, x(s))-f(s, \gamma(s))| d s \\
& \leq \frac{L}{\Gamma(q+1)}\left(1+\frac{\Delta_{2}|\sigma|\left(\beta^{q+1}-\alpha^{q+1}\right)+\Delta_{1}|\eta|\left(\delta^{q+1}-\gamma^{q+1}\right)+(q+1) \Delta_{1}}{(q+1)|\Delta|}\right) \| x-\gamma|| \\
& =L \Lambda|| x-\gamma||,
\end{aligned}
$$


where $\Lambda$ is given by (3.2). Observe that $\Lambda$ depends only on the parameters involved in the problem. As $L<1 / \Lambda$, therefore $\mathbf{F}$ is a contraction. Thus, the conclusion of the theorem follows by the contraction mapping principle (Banach fixed point theorem).

Now, we prove the existence of solutions of (1.1) by applying Krasnoselskii's fixed point theorem [27].

Theorem 3.2 (Krasnoselskii's fixed point theorem). Let $M$ be a closed, bounded, convex, and nonempty subset of a Banach space X. Let $A, B$ be the operators such that (i) $A x+B y \in M$ whenever $x, y \in M$; (ii) $A$ is compact and continuous; (iii) $B$ is a contraction mapping. Then there exists $z \in M$ such that $z=A z+B z$.

Theorem 3.3 Let $f:[0,1] \times \mathbb{R} \rightarrow \mathbb{R}$ be a jointly continuous function satisfying the assumptions $\left(A_{1}\right)$ and $\left(A_{2}\right)$ with

$$
\frac{L}{\Gamma(q+1)}\left(\frac{\Delta_{2}|\sigma|\left(\beta^{q+1}-\alpha^{q+1}\right)+\Delta_{1}|\eta|\left(\delta^{q+1}-\gamma^{q+1}\right)+(q+1) \Delta_{1}}{(q+1)|\Delta|}\right)<1 .
$$

Then the boundary value problem (1.1) has at least one solution on $[0,1]$.

Proof. Letting $\sup _{t \in[0,1]}|\mu(t)|=\|\mu\|$, we fix

$$
\bar{r} \geq \frac{\|\mu\|}{\Gamma(q+1)}\left(1+\frac{\Delta_{2}|\sigma|\left(\beta^{q+1}-\alpha^{q+1}\right)+\Delta_{1}|\eta|\left(\delta^{q+1}-\gamma^{q+1}\right)+(q+1) \Delta_{1}}{(q+1)|\Delta|}\right),
$$

and consider $B_{\bar{r}}=\{x \in \mathcal{C}:\|x\| \leq \bar{r}\}$. We define the operators $\mathcal{P}$ and $\mathcal{Q}$ on $B_{\bar{r}}$ as

$$
(\mathcal{P} x)(t)=\int_{0}^{t} \frac{(t-s)^{q-1}}{\Gamma(q)} f(s, x(s)) d s,
$$

$(\mathcal{Q} x)(t)$

$$
\begin{aligned}
= & \frac{\sigma}{\Delta \Gamma(q)}\left[-\left(\frac{\eta}{2}\left(\delta^{2}-\gamma^{2}\right)-1\right)+t(\eta(\delta-\gamma)-1)\right] \int_{\alpha}^{\beta}\left(\int_{0}^{s}(s-m)^{q-1} f(m, x(m)) d m\right) d s \\
& +\frac{\eta}{\Delta \Gamma(q)}\left[\frac{\sigma}{2}\left(\beta^{2}-\alpha^{2}\right)-(\sigma(\beta-\alpha)-1) t\right] \int_{\gamma}^{\delta}\left(\int_{0}^{s}(s-m)^{q-1} f(m, x(m)) d m\right) d s \\
& -\frac{1}{\Delta \Gamma(q)}\left[\frac{\sigma}{2}\left(\beta^{2}-\alpha^{2}\right)-(\sigma(\beta-\alpha)-1) t\right] \int_{0}^{1}(1-s)^{q-1} f(s, x(s)) d s .
\end{aligned}
$$

For $x, y \in B_{\bar{r}}$, we find that

$$
\begin{aligned}
& \|\mathcal{P} x+\mathcal{Q} y\| \\
& \leq \frac{\|\mu\|}{\Gamma(q+1)}\left(1+\frac{\Delta_{2}|\sigma|\left(\beta^{q+1}-\alpha^{q+1}\right)+\Delta_{1}|\eta|\left(\delta^{q+1}-\gamma^{q+1}\right)+(q+1) \Delta_{1}}{(q+1)|\Delta|}\right) \leq \bar{r} .
\end{aligned}
$$

Thus, $\mathcal{P} x+\mathcal{Q} y \in B_{\bar{r}}$. It follows from the assumption $\left(A_{1}\right)$ together with (3.3) that $\mathcal{Q}$ is a contraction mapping. Continuity of $f$ implies that the operator $\mathcal{P}$ is continuous. Also, $\mathcal{P}$ is uniformly bounded on $B_{\bar{r}}$ as

$$
\|\mathcal{P} x\| \leq \frac{\|\mu\|}{\Gamma(q+1)} .
$$

Now we prove the compactness of the operator $\mathcal{P}$. 
In view of $\left(A_{1}\right)$, we define $\sup _{(t, x) \in[0,1] \times B_{\bar{\tau}}} \mid f(t, x)=\bar{f}$, and consequently we have

$$
\begin{aligned}
\left|(\mathcal{P} x)\left(t_{1}\right)-(\mathcal{P} x)\left(t_{2}\right)\right|= & \mid \frac{1}{\Gamma(q)} \int_{0}^{t_{1}}\left[\left(t_{2}-s\right)^{q-1}-\left(t_{1}-s\right)^{q-1}\right] f(s, x(s)) d s \\
& +\int_{t_{1}}^{t_{2}}\left(t_{2}-s\right)^{q-1} f(s, x(s)) d s \mid \\
\leq & \frac{\bar{f}}{\Gamma(q+1)}\left|2\left(t_{2}-t_{1}\right)^{q}+t_{1}^{q}-t_{2}^{q}\right|
\end{aligned}
$$

which is independent of $x$. Thus, $\mathcal{P}$ is equicontinuous. Hence, by the Arzelá-Ascoli Theorem, $\mathcal{P}$ is compact on $B_{\bar{r}}$. Thus all the assumptions of Theorem 3.2 are satisfied. So the conclusion of Theorem 3.2 implies that the boundary value problem (1.1) has at least one solution on $[0,1]$.

Our next existence result is based on Leray-Schauder degree theory.

Theorem 3.4 Let $f:[0,1] \times \mathbb{R} \rightarrow \mathbb{R}$. Assume that there exist constants $0 \leq \kappa<\frac{1}{\Lambda}$, where $\Lambda$ is given by (3.2) and $M>0$ such that $|f(t, x)| \leq \kappa|x|+M$ for all $t \in$ $[0,1], x \in C[0,1]$. Then the boundary value problem (1.1) has at least one solution.

Proof. Consider the fixed point problem

$$
x=\mathbf{F} x,
$$

where $\mathbf{F}$ is defined by (3.1). In view of the fixed point problem (3.4), we just need to prove the existence of at least one solution $x \in C[0,1]$ satisfying (3.4). Define a suitable ball $B_{R} \subset C[0,1]$ with radius $R>0$ as

$$
B_{R}=\left\{x \in C[0,1]: \max _{t \in[0,1]}|x(t)|<R\right\},
$$

where $R$ will be fixed later. Then, it is sufficient to show that $\mathbf{F}: \bar{B}_{R} \rightarrow C[0,1]$ satisfies

$$
x \neq \lambda \mathbf{F} x, \quad \forall x \in \partial B_{R} \quad \text { and } \quad \forall \lambda \in[0,1] .
$$

Let us set

$$
H(\lambda, x)=\lambda \mathbf{F} x, \quad x \in C(\mathbb{R}) \quad \lambda \in[0,1] .
$$

Then, by the Arzelá-Ascoli Theorem, $h_{\lambda}(x)=x-H(\lambda, x)=x-\lambda \mathbf{F} x$ is completely continuous. If (3.5) is true, then the following Leray-Schauder degrees are well defined and by the homotopy invariance of topological degree, it follows that

$$
\begin{aligned}
\operatorname{deg}\left(h_{\lambda}, B_{R}, 0\right) & =\operatorname{deg}\left(I-\lambda F, B_{R}, 0\right)=\operatorname{deg}\left(h_{1}, B_{R}, 0\right) \\
& =\operatorname{deg}\left(h_{0}, B_{R}, 0\right)=\operatorname{deg}\left(I, B_{R}, 0\right)=1 \neq 0,0 \in B_{r},
\end{aligned}
$$

where $I$ denotes the unit operator. By the nonzero property of Leray-Schauder degree, $h_{1}(t)=x-\lambda \mathbf{F} x=0$ for at least one $x \in B_{R}$. In order to prove (3.5), we assume that $x=\lambda \mathbf{F} x, \lambda \in[0,1]$. Then for $x \in \partial B_{R}$ and $t \in[0,1]$ we have 


$$
\begin{aligned}
|x(t)|=|\lambda(\mathbf{F} x)(t)| & \frac{1}{\Gamma(q)} \int_{0}^{t}(t-s)^{q-1}|f(s, x(s))| d s \\
& +\mid \frac{\sigma}{\Delta \Gamma(q)}\left[-\left(\frac{\eta}{2}\left(\delta^{2}-\gamma^{2}\right)-1\right)+t(\eta(\delta-\gamma)-1] \mid \int_{\alpha}^{\beta}\left(\int_{0}^{s}(s-m)^{q-1}|f(m, x(m))| d m\right) d s\right. \\
& +\left|\frac{\eta}{\Delta \Gamma(q)}\left[\frac{\sigma}{2}\left(\beta^{2}-\alpha^{2}\right)-(\sigma(\beta-\alpha)-1) t\right]\right| \int_{\gamma}^{\delta}\left(\int_{0}^{s}(s-m)^{q-1}|f(m, x(m))| d m\right) d s \\
& +\left|\frac{1}{\Delta \Gamma(q)}\left[\frac{\sigma}{2}\left(\beta^{2}-\alpha^{2}\right)-(\sigma(\beta-\alpha)-1) t\right]\right| \int_{0}^{1}(1-s)^{q-1}|f(s, x(s))| d s \\
\leq & (\kappa|| x||+M)\left[\frac{1}{\Gamma(q)} \int_{0}^{1}(1-s)^{q-1} d s+\frac{|\sigma|}{|\Delta| \Gamma(q)} \Delta_{2} \int_{\alpha}^{s}\left(\int_{0}^{s}(s-m)^{q-1} d m\right) d s\right. \\
& \left.+\frac{|\eta|}{|\Delta| \Gamma(q)} \Delta_{1} \int_{\gamma}^{\delta}\left(\int_{0}^{s}(s-m)^{q-1} d m\right) d s+\frac{1}{|\Delta| \Gamma(q)} \Delta_{1} \int_{0}^{1}(1-s)^{q-1} d s\right] \\
\leq & \frac{\kappa \| x||+M}{\Gamma(q+1)}\left(1+\frac{\Delta_{2}|\sigma|\left(\beta^{q+1}-\alpha^{q+1}\right)+\Delta_{1}|\eta|\left(\delta^{q+1}-\gamma^{q+1}\right)+(q+1) \Delta_{1}}{(q+1)|\Delta|}\right) \\
= & (\kappa\|x\|+M) \Lambda,
\end{aligned}
$$

which, on taking norm $\left(\sup _{t \in[0,1]}|x(t)|=\|x\|\right)$ and solving for $\|x\|$, yields

$$
\|x\| \leq \frac{M \Lambda}{1-\kappa \Lambda} .
$$

Letting $R=\frac{M \Lambda}{1-\kappa \Lambda}+1,(3.5)$ holds. This completes the proof. $\square$

Example 3.5 Consider the following strip fractional boundary value problem

$$
\left\{\begin{array}{c}
{ }^{c} D^{3 / 2} x(t)=\frac{1}{(t+2)^{2}} \frac{|x|}{1+|x|}, t \in[0,1] \\
x(0)=\int_{1 / 3}^{1 / 2} x(s) d s, x(1)=\int_{2 / 3}^{3 / 4} x(s) d s .
\end{array}\right.
$$

Here, $q=3 / 2, \sigma=1, \eta=1, \alpha=1 / 3, \beta=1 / 2, \gamma=2 / 3, \delta=3 / 4$ and $f(t, x)=\frac{1}{(t+2)^{2}} \frac{|x|}{1+|x|}$. As $|f(t, x)-f(t, y)| \leq \frac{1}{4}|x-y|$, therefore, $\left(A_{1}\right)$ is satisfied with $L=\frac{1}{4}$. Further, $\Delta_{1}=65 / 72, \Delta_{2}=535 / 288, \Delta=4945 / 5184$, and

$$
\Lambda=\frac{1}{\Gamma(q+1)}\left(1+\frac{\Delta_{2}|\sigma|\left(\beta^{q+1}-\alpha^{q+1}\right)+\Delta_{1}|\eta|\left(\delta^{q+1}-\gamma^{q+1}\right)+(q+1) \Delta_{1}}{(q+1)|\Delta|}\right)=1.128765 .
$$

Clearly, $L \Lambda=0.282191<1$. Thus, by the conclusion of Theorem 3.1, the boundary value problem (3.6) has a unique solution on $[0,1]$.

Example 3.6 Consider the following boundary value problem

$$
\left\{\begin{array}{l}
{ }^{c} D^{3 / 2} x(t)=\frac{1}{(4 \pi)} \sin (2 \pi x)+\frac{|x|}{1+|x|}, \quad t \in[0,1], \quad 1<q \leq 2, \\
x(0)=\int_{1 / 3}^{1 / 2} x(s) d s, \quad x(1)=\int_{2 / 3}^{3 / 4} x(s) d s .
\end{array}\right.
$$


Here,

$$
|f(t, x)|=\left|\frac{1}{(4 \pi)} \sin (2 \pi x)+\frac{|x|}{1+|x|}\right| \leq \frac{1}{2}|x|+1
$$

Clearly $M=1$ and

$$
\kappa=\frac{1}{2}<\frac{1}{\Lambda}=0.885924
$$

Thus, all the conditions of Theorem 3.4 are satisfied and consequently the problem (3.7) has at least one solution.

\section{Existence results for multi-valued case}

\subsection{Preliminaries}

Let us recall some basic definitions on multi-valued maps [28,29].

For a normed space $(X,\|\|$.$) , let P_{c l}(X)=\{Y \in \mathcal{P}(X): Y$ is closed $\}$, $P_{c p}(X)=\{Y \in \mathcal{P}(X): Y$ is compact $\}, \quad P_{c p}(X)=\{Y \in \mathcal{P}(X): Y$ is compact $\}, \quad$ and $P_{c p, c}(X)=\{Y \in \mathcal{P}(X): Y$ is compact and convex $\}$. A multi-valued map $G: X \rightarrow \mathcal{P}(X)$ is convex (closed) valued if $G(x)$ is convex (closed) for all $x \in X$. The map $G$ is bounded on bounded sets if $G(\mathbb{B})=\cup_{x \in \mathbb{B}} G(x)$ is bounded in $X$ for all $\mathbb{B} \in P_{b}(X)$ (i.e., $\left.\sup _{x \in \mathbb{B}}\{\sup \{|y|: y \in G(x)\}\}<\infty\right)$ ). $G$ is called upper semi-continuous (u.s.c.) on $X$ if for each $x_{0} \in X$, the set $G\left(x_{0}\right)$ is a nonempty closed subset of $X$, and if for each open set $N$ of $X$ containing $G\left(x_{0}\right)$, there exists an open neighborhood $\mathcal{N}_{0}$ of $x_{0}$ such that $G\left(\mathcal{N}_{0}\right) \subseteq N$. G is said to be completely continuous if $G(\mathbb{B})$ is relatively compact for every $\mathbb{B} \in P_{b}(X)$. If the multi-valued map $G$ is completely continuous with nonempty compact values, then $G$ is u.s.c. if and only if $G$ has a closed graph, i.e., $x_{n} \rightarrow x_{*}, y_{n} \rightarrow y_{*}, y_{n} \in G\left(x_{n}\right)$ imply $y_{*} \in G\left(x_{*}\right)$. G has a fixed point if there is $x \in X$ such that $x \in G(x)$. The fixed point set of the multivalued operator $G$ will be denoted by Fix $G$. A multivalued map $G:[0 ; 1] \rightarrow P_{c l}(\mathbb{R})$ is said to be measurable if for every $y \in \mathbb{R}$, the function

$$
t \mapsto d(y, G(t))=\inf \{|y-z|: z \in G(t)\}
$$

is measurable.

Let $C([0,1])$ denotes a Banach space of continuous functions from $[0,1]$ into $\mathbb{R}$ with the norm $\|x\|=\sup _{t \in[0,1]}|x(t)|$. Let $L^{1}([0,1], \mathbb{R})$ be the Banach space of measurable functions $x:[0,1] \rightarrow \mathbb{R}$ which are Lebesgue integrable and normed by $\|x\|_{L^{1}}=\int_{0}^{1}|x(t)| d t$.

Definition 4.1 A multivalued map $F:[0,1] \times \mathbb{R} \rightarrow \mathcal{P}(\mathbb{R})$ is said to be Carathéodory if

(i) $t \mapsto F(t, x)$ is measurable for each $x \in \mathbb{R}$;

(ii) $x \mapsto F(t, x)$ is upper semicontinuous for almost all $t \in[0,1]$;

Further a Carathéodory function $F$ is called $L^{1}$-Carathéodory if 
(iii) for each $\alpha>0$, there exists $\varphi_{\alpha} \in L^{1}\left([0,1], \mathbb{R}^{+}\right)$such that

$$
\|F(t, x)\|=\sup \{|v|: v \in F(t, x)\} \leq \varphi_{\alpha}(t)
$$

for all $\|x\|_{\infty} \leq \alpha$ and for a. e. $t \in[0,1]$.

For each $y \in C([0,1], \mathbb{R})$, define the set of selections of $F$ by

$$
S_{F, y}:=\left\{v \in L^{1}([0,1], \mathbb{R}): v(t) \in F(t, \gamma(t)) \text { for a.e. } t \in[0,1]\right\} .
$$

Let $X$ be a nonempty closed subset of a Banach space $E$ and $G: X \rightarrow \mathcal{P}(E)$ be a multivalued operator with nonempty closed values. $G$ is lower semi-continuous (l.s.c.) if the set $\{y \in X: G(y) \cap B \neq \varnothing\}$ is open for any open set $B$ in $E$. Let $A$ be a subset of $[0,1] \times \mathbb{R} . A$ is $\mathcal{L} \otimes \mathcal{B}$ measurable if $A$ belongs to the $\sigma$-algebra generated by all sets of the form $\mathcal{J} \times \mathcal{D}$, where $\mathcal{J}$ is Lebesgue measurable in $[0,1]$ and $\mathcal{D}$ is Borel measurable in $\mathbb{R}$. A subset $\mathcal{A}$ of $L^{1}([0,1], \mathbb{R})$ is decomposable if for all $u, v \in \mathcal{A}$ and measurable $\mathcal{J} \subset[0,1]=J$, the function $u \chi_{\mathcal{J}}+v \chi_{\mathcal{J}-\mathcal{J}} \in \mathcal{A}$, where $\chi_{\mathcal{J}}$ stands for the characteristic function of $\mathcal{J}$.

Definition 4.2 Let $Y$ be a separable metric space and let $N: Y \rightarrow \mathcal{P}\left(L^{1}([0,1], \mathbb{R})\right)$ be a multivalued operator. We say $N$ has a property $(B C)$ if $N$ is lower semi-continuous (l.s.c.) and has nonempty closed and decomposable values.

Let $F:[0,1] \times \mathbb{R} \rightarrow \mathcal{P}(\mathbb{R})$ be a multivalued map with nonempty compact values. Define a multivalued operator $\mathcal{F}: C([0,1] \times \mathbb{R}) \rightarrow \mathcal{P}\left(L^{1}([0,1], \mathbb{R})\right)$ associated with $F$ as

$$
\mathcal{F}(x)=\left\{w \in L^{1}([0,1], \mathbb{R}): w(t) \in F(t, x(t)) \text { for a.e. } t \in[0,1]\right\},
$$

which is called the Nemytskii operator associated with $F$.

Definition 4.3 Let $F:[0,1] \times \mathbb{R} \rightarrow \mathcal{P}(\mathbb{R})$ be a multivalued function with nonempty compact values. We say $F$ is of lower semi-continuous type (l.s.c. type) if its associated Nemytskii operator $\mathcal{F}$ is lower semi-continuous and has nonempty closed and decomposable values.

Let $(X, d)$ be a metric space induced from the normed space $(X ;\|\|$.$) . Consider$ $H_{d}: \mathcal{P}(X) \times \mathcal{P}(X) \rightarrow \mathbb{R} \cup\{\infty\}$ given by

$$
H_{d}(A, B)=\max \left\{\sup _{a \in A} d(a, B), \sup _{b \in B} d(A, b)\right\},
$$

where $d(A, b)=\inf _{a \in A} d(a ; b)$ and $d(a, B)=\inf _{b \in B} d(a ; b)$. Then $\left(P_{b, c l}(X), H_{d}\right)$ is a metric space and $\left(P_{c l}(X), H_{d}\right)$ is a generalized metric space (see [30]).

Definition 4.4 A multivalued operator $N: X \rightarrow P_{c l}(X)$ is called:

(a) $\gamma$-Lipschitz if and only if there exists $\gamma>0$ such that

$$
H_{d}(N(x), N(y)) \leq \gamma d(x, y) \text { for each } x, y \in X ;
$$

(b) a contraction if and only if it is $\gamma$-Lipschitz with $\gamma<1$.

The following lemmas will be used in the sequel.

Lemma 4.5 (Nonlinear alternative for Kakutani maps) [31]. Let E be a Banach space, $C$ is a closed convex subset of $E, U$ is an open subset of $C$ and $0 \in U$. Suppose 
that $F: \bar{U} \rightarrow \mathcal{P}_{c, c v}(C)$ is a upper semicontinuous compact map; here $\mathcal{P}_{c, c v}(C)$ denotes the family of nonempty, compact convex subsets of $C$. Then either

(i) F has a fixed point in $\bar{U}$, or

(ii) there is $a u \in \partial U$ and $\lambda \in(0,1)$ with $u \in \lambda F(u)$.

Lemma 4.6 [32]Let $X$ be a Banach space. Let $F:[0, T] \times \mathbb{R} \rightarrow \mathcal{P}_{c p, c}(X)$ be an $L^{1}$ Carathéodory multivalued map and let $\theta$ be a linear continuous mapping from $L^{1}([0$, $1], X)$ to $C([0,1], X)$. Then the operator

$$
\Theta \circ S_{F}: C([0,1], X) \rightarrow P_{c p, c}(C([0,1], X)), \quad x \mapsto\left(\Theta \circ S_{F}\right)(x)=\Theta\left(S_{F, x}\right)
$$

is a closed graph operator in $C([0,1], X) \times C([0,1], X)$.

Lemma 4.7 [33]Let $Y$ be a separable metric space and let $N: Y \rightarrow \mathcal{P}\left(L^{1}([0,1], \mathbb{R})\right)$ be a multivalued operator satisfying the property $(B C)$. Then $N$ has a continuous selection, that is, there exists a continuous function (single-valued) $g: Y \rightarrow L^{1}([0,1], \mathbb{R})$ such that $g(x) \in N(x)$ for every $x \in Y$.

Lemma 4.8 [34] Let $(X, d)$ be a complete metric space. If $N: X \rightarrow P_{c l}(X)$ is a contraction, then $\operatorname{FixN} \neq \varnothing$.

Definition 4.9 A function $x \in C^{2}([0,1], \mathbb{R})$ is a solution of the problem (1.2) if $x(0)=\sigma \int_{\alpha}^{\beta} x(s) d s, x(1)=\eta \int_{\gamma}^{\delta} x(s) d s$, and there exists a function $f \in L^{1}([0,1], \mathbb{R})$ such that

$$
\begin{aligned}
& f(t) \in F(t, x(t)) \text { a.e. on }[0,1] \text { and } \\
& \qquad \begin{aligned}
x(t) & =\frac{1}{\Gamma(q)} \int_{0}^{t}(t-s)^{q-1} f(s) d s \\
& +\frac{\sigma}{\Delta \Gamma(q)}\left[-\left(\frac{\eta}{2}\left(\delta^{2}-\gamma^{2}\right)-1\right)+t(\eta(\delta-\gamma)-1)\right] \int_{\alpha}^{\beta}\left(\int_{0}^{s}(s-m)^{q-1} f(m) d m\right) d s \\
& +\frac{\eta}{\Delta \Gamma(q)}\left[\frac{\sigma}{2}\left(\beta^{2}-\alpha^{2}\right)-(\sigma(\beta-\alpha)-1) t\right] \int_{\gamma}^{\delta}\left(\int_{0}^{s}(s-m)^{q-1} f(m) d m\right) d s \\
& -\frac{1}{\Delta \Gamma(q)}\left[\frac{\sigma}{2}\left(\beta^{2}-\alpha^{2}\right)-(\sigma(\beta-\alpha)-1) t\right] \int_{0}^{1}(1-s)^{q-1} f(s) d s .
\end{aligned}
\end{aligned}
$$

\subsection{The Carathéodory case}

Theorem 4.10 Assume that:

$\left(H_{1}\right) F:[0,1] \times \mathbb{R} \rightarrow \mathcal{P}(\mathbb{R})$ is Carathéodory and has nonempty compact and convex values;

$\left(H_{2}\right)$ there exists a continuous nondecreasing function $\psi:[0, \infty) \rightarrow(0, \infty)$ and a function $p \in L^{1}\left([0,1], \mathbb{R}^{+}\right)$such that

$$
\|F(t, x)\|_{\mathcal{P}}:=\sup \{|y|: y \in F(t, x)\} \leq p(t) \psi(\| x||) \quad \text { for each } \quad(t, x) \in[0,1] \times \mathbb{R} .
$$


$\left(H_{3}\right)$ there exists a constant $M>0$ such that

$$
\begin{aligned}
& M \cdot\left\{\frac { \psi ( M ) } { \Gamma ( q ) } \left\{\left(1+\frac{\Delta_{1}}{|\Delta|}\right) \int_{0}^{1} p(s) d s+\frac{|\sigma| \Delta_{2}}{|\Delta|} \int_{\alpha}^{\beta}\left(\int_{0}^{s}(s-m)^{q-1} p(m) d m\right) d s\right.\right. \\
& \left.\left.+\frac{|\eta| \Delta_{1}}{|\Delta|} \int_{\gamma}^{\delta}\left(\int_{0}^{s}(s-m)^{q-1} p(m) d m\right) d s\right\}\right\}^{-1}>1 .
\end{aligned}
$$

Then the boundary value problem (1.2) has at least one solution on [0, 1].

Proof. Define the operator $\Omega_{F}: C([0,1], \mathbb{R}) \rightarrow \mathcal{P}(C([0,1], \mathbb{R}))$ by

$$
\Omega_{F}(x)=\left\{\begin{array}{l}
h \in C([0,1], \mathbb{R}): \\
h(t)=\left\{\begin{array}{l}
\frac{1}{\Gamma(q)} \int_{0}^{t}(t-s)^{q-1} f(s) d s \\
+\frac{\sigma}{\Delta \Gamma(q)}\left[-\left(\frac{\eta}{2}\left(\delta^{2}-\gamma^{2}\right)-1\right)+t(\eta(\delta-\gamma)-1)\right] \times \\
\times \int_{\alpha}^{\beta}\left(\int_{0}^{s}(s-m)^{q-1} f(m) d m\right) d s \\
+\frac{\eta}{\Delta \Gamma(q)}\left[\frac{\sigma}{2}\left(\beta^{2}-\alpha^{2}\right)-(\sigma(\beta-\alpha)-1) t\right] \times \\
\times \int_{\gamma}^{\delta}\left(\int_{0}^{s}(s-m)^{q-1} f(m) d m\right) d s \\
-\frac{1}{\Delta \Gamma(q)}\left[\frac{\sigma}{2}\left(\beta^{2}-\alpha^{2}\right)-(\sigma(\beta-\alpha)-1) t\right] \int_{0}^{1}(1-s)^{q-1} f(s) d s
\end{array}\right.
\end{array}\right.
$$

for $f \in S_{F, x}$. We will show that $\Omega_{F}$ satisfies the assumptions of the nonlinear alternative of Leray-Schauder type. The proof consists of several steps. As a first step, we show that $\Omega_{F}$ is convex for each $x \in C([0,1], \mathbb{R})$. This step is obvious since $S_{F, x}$ is convex ( $F$ has convex values), and therefore we omit the proof.

In the second step, we show that $\Omega_{F}$ maps bounded sets (balls) into bounded sets in $C([0,1], \mathbb{R})$. For a positive number $\rho$, let $B_{\rho}=\{x \in C([0,1], \mathbb{R}):\|x\| \leq \rho\}$ be a bounded ball in $C([0,1], \mathbb{R})$. Then, for each $h \in \Omega_{F}(x), x \in B_{\rho}$, there exists $f \in S_{F, x}$ such that

$$
\begin{aligned}
& h(t)=\frac{1}{\Gamma(q)} \int_{0}^{t}(t-s)^{q-1} f(s) d s \\
& +\frac{\sigma}{\Delta \Gamma(q)}\left[-\left(\frac{\eta}{2}\left(\delta^{2}-\gamma^{2}\right)-1\right)+t(\eta(\delta-\gamma)-1)\right] \int_{\alpha}^{\beta}\left(\int_{0}^{s}(s-m)^{q-1} f(m) d m\right) d s \\
& +\frac{\eta}{\Delta \Gamma(q)}\left[\frac{\sigma}{2}\left(\beta^{2}-\alpha^{2}\right)-(\sigma(\beta-\alpha)-1) t\right] \int_{\gamma}^{\delta}\left(\int_{0}^{s}(s-m)^{q-1} f(m) d m\right) d s \\
& -\frac{1}{\Delta \Gamma(q)}\left[\frac{\sigma}{2}\left(\beta^{2}-\alpha^{2}\right)-(\sigma(\beta-\alpha)-1) t\right] \int_{0}^{1}(1-s)^{q-1} f(s) d s .
\end{aligned}
$$


Then for $t \in[0,1]$ we have

$$
\begin{aligned}
|h(t)| \leq & \frac{1}{\Gamma(q)} \int_{0}^{t}(t-s)^{q-1}|f(s, x(s))| d s \\
& +\left|\frac{\sigma}{\Delta \Gamma^{\mathrm{t}}(q)}\left[-\left(\frac{\eta}{2}\left(\delta^{2}-\gamma^{2}\right)-1\right)+t(\eta(\delta-\gamma)-1)\right]\right| \int_{\alpha}^{\beta}\left(\int_{0}^{s}(s-m)^{q-1} f(m) d m\right) d s \\
& +\left|\frac{\eta}{\Delta \Gamma(q)}\left[\frac{\sigma}{2}\left(\beta^{2}-\alpha^{2}\right)-(\sigma(\beta-\alpha)-1) t\right]\right| \int_{\gamma}^{\delta}\left(\int_{0}^{s}(s-m)^{q-1} f(m) d m\right) d s \\
& +\left|\frac{1}{\Delta \Gamma(q)}\left[\frac{\sigma}{2}\left(\beta^{2}-\alpha^{2}\right)-(\sigma(\beta-\alpha)-1) t\right]\right| \int_{0}^{1}(1-s)^{q-1} f(s) d s \\
\leq & \psi(\|x\|)\left[\frac{1}{\Gamma(q)} \int_{0}^{t}(t-s)^{q-1} p(s) d s+\frac{|\sigma|}{|\Delta| \Gamma(q)} \Delta_{2} \int_{\alpha}^{\beta}\left(\int_{0}^{s}(s-m)^{q-1} p(m) d m\right) d s\right. \\
& \left.+\frac{|\eta|}{|\Delta| \Gamma(q)} \Delta_{1} \int_{\gamma}^{\delta}\left(\int_{0}^{s}(s-m)^{q-1} p(m) d m\right) d s+\frac{1}{|\Delta| \Gamma(q)} \Delta_{1}^{1} \int_{0}^{1}(1-s)^{q-1} p(s) d s\right] \\
\leq & \frac{\psi(\|x\|)}{\Gamma(q)}\left\{\left(1+\frac{\Delta_{1}}{|\Delta|}\right) \int_{0}^{1} p(s) d s+\frac{|\sigma| \Delta_{2}}{|\Delta|} \int_{\alpha}^{\beta}\left(\int_{0}^{s}(s-m)^{q-1} f(m) d m\right) d s\right. \\
& \left.+\frac{|\eta| \Delta_{1}}{|\Delta|} \int_{\gamma}^{\delta}\left(\int_{0}^{s}(s-m)^{q-1} p(m) d m\right) d s\right\} .
\end{aligned}
$$

Thus,

$$
\begin{aligned}
\|h\| \leq & \frac{\psi(\rho)}{\Gamma(q)}\left\{\left(1+\frac{\Delta_{1}}{|\Delta|}\right) \int_{0}^{1} p(s) d s+\frac{|\sigma| \Delta_{2}}{|\Delta|} \int_{\alpha}^{\beta}\left(\int_{0}^{s}(s-m)^{q-1} p(m) d m\right) d s\right. \\
& \left.+\frac{|\eta| \Delta_{1}}{|\Delta|} \int_{\gamma}^{\delta}\left(\int_{0}^{s}(s-m)^{q-1} p(m) d m\right) d s\right\} .
\end{aligned}
$$

Now we show that $\Omega_{F}$ maps bounded sets into 'equicontinuous sets of $C([0,1], \mathbb{R})$. Let $t^{\prime}, t^{\prime \prime} \in[0,1]$ with $t^{\prime}<t^{\prime \prime}$ and $x \in B_{\rho}$. For each $h \in \Omega_{F}(x)$, we obtain

$$
\begin{aligned}
& \left|h\left(t^{\prime \prime}\right)-h\left(t^{\prime}\right)\right| \\
& \leq\left|\psi(\|x\|) \int_{0}^{t^{\prime}}\left[\frac{\left(t^{\prime \prime}-s\right)^{q-1}-\left(t^{\prime}-s\right)^{q-1}}{\Gamma(q)}\right] p(s) d s+\psi(\|x\|) \int_{t^{\prime}}^{t^{\prime \prime}} \frac{\left(t^{\prime \prime}-s\right)^{q-1}}{\Gamma(q)} p(s) d s\right| \\
& +\left|\frac{\psi(\|x\|) \sigma}{\Delta \Gamma(q)}(\eta(\delta-\gamma)-1)\right| t^{\prime \prime}-t^{\prime}|| \int_{\alpha}^{\beta}\left(\int_{0}^{s}(s-m)^{q-1} p(m) d m\right) d s \\
& +\left|\frac{\eta \psi(\|x\|)}{\Delta \Gamma(q)}\left[(\sigma(\beta-\alpha)+1)\left|t^{\prime \prime}-t^{\prime}\right|\right]\right| \int_{\gamma}^{\delta}\left(\int_{0}^{s}(s-m)^{q-1} p(m) d m\right) d s \\
& +\left|\frac{\psi(\|x\|)}{\Delta \Gamma(q)}\left[(\sigma(\beta-\alpha)+1)\left|t^{\prime \prime}-t^{\prime}\right|\right]\right| \int_{0}^{1}(1-s)^{q-1} p(s) d s .
\end{aligned}
$$

Obviously the right-hand side of the above inequality tends to zero independently of $x \in B_{\rho}$ as $t^{\prime \prime}-t^{\prime} \rightarrow 0$. As $\Omega_{F}$ satisfies the above three assumptions, therefore it follows by the Ascoli-Arzelá theorem that $\Omega_{F}: C([0,1], \mathbb{R}) \rightarrow \mathcal{P}(C([0,1], \mathbb{R}))$ is completely continuous. 
In our next step, we show that $\Omega_{F}$ has a closed graph. Let $x_{n} \rightarrow x_{*}, h_{n} \in \Omega_{F}\left(x_{n}\right)$, and $h_{n} \rightarrow h_{*}$. Then we need to show that $h_{*} \in \Omega_{F}\left(x_{*}\right)$. Associated with $h_{n} \in \Omega_{F}$ $\left(x_{n}\right)$, there exists $f_{n} \in S_{F, x_{n}}$ such that for each $t \in[0,1]$,

$$
\begin{aligned}
h_{n}(t)= & \frac{1}{\Gamma(q)} \int_{0}^{t}(t-s)^{q-1} f_{n}(s) d s \\
& +\frac{\sigma}{\Delta \Gamma(q)}\left[-\left(\frac{\eta}{2}\left(\delta^{2}-\gamma^{2}\right)-1\right)+t(\eta(\delta-\gamma)-1)\right] \int_{\alpha}^{\beta}\left(\int_{0}^{s}(s-m)^{q-1} f_{n}(m) d m\right) d s \\
& +\frac{\eta}{\Delta \Gamma(q)}\left[\frac{\sigma}{2}\left(\beta^{2}-\alpha^{2}\right)-(\sigma(\beta-\alpha)-1) t\right] \int_{\gamma}^{\delta}\left(\int_{0}^{s}(s-m)^{q-1} f_{n}(m) d m\right) d s \\
& -\frac{1}{\Delta \Gamma(q)}\left[\frac{\sigma}{2}\left(\beta^{2}-\alpha^{2}\right)-(\sigma(\beta-\alpha)-1) t\right] \int_{0}^{1}(1-s)^{q-1} f_{n}(s) d s,
\end{aligned}
$$

Thus it suffices to show that there exists $f_{*} \in S_{F, x_{*}}$ such that for each $t \in[0,1]$,

$$
\begin{aligned}
h_{*}(t)= & \frac{1}{\Gamma(q)} \int_{0}^{t}(t-s)^{q-1} f_{*}(s) d s \\
& +\frac{\sigma}{\Delta \Gamma(q)}\left[-\left(\frac{\eta}{2}\left(\delta^{2}-\gamma^{2}\right)-1\right)+t(\eta(\delta-\gamma)-1)\right] \int_{\alpha}^{\beta}\left(\int_{0}^{s}(s-m)^{q-1} f_{*}(m) d m\right) d s \\
& +\frac{\eta}{\Delta \Gamma(q)}\left[\frac{\sigma}{2}\left(\beta^{2}-\alpha^{2}\right)-(\sigma(\beta-\alpha)-1) t\right] \int_{\gamma}^{\delta}\left(\int_{0}^{s}(s-m)^{q-1} f_{*}(m) d m\right) d s \\
& -\frac{1}{\Delta \Gamma(q)}\left[\frac{\sigma}{2}\left(\beta^{2}-\alpha^{2}\right)-(\sigma(\beta-\alpha)-1) t\right] \int_{0}^{1}(1-s)^{q-1} f_{*}(s) d s,
\end{aligned}
$$

Let us consider the linear operator $\theta: L^{1}([0,1], \mathbb{R}) \rightarrow C([0,1], \mathbb{R})$ given by

$$
\begin{aligned}
f \mapsto \Theta(f)(t)= & \frac{1}{\Gamma(q)} \int_{0}^{t}(t-s)^{q-1} f(s) d s \\
& +\frac{\sigma}{\Delta \Gamma(q)}\left[-\left(\frac{\eta}{2}\left(\delta^{2}-\gamma^{2}\right)-1\right)+t(\eta(\delta-\gamma)-1)\right] \int_{\alpha}^{\beta}\left(\int_{0}^{s}(s-m)^{q-1} f(m) d m\right) d s \\
& +\frac{\eta}{\Delta \Gamma(q)}\left[\frac{\sigma}{2}\left(\beta^{2}-\alpha^{2}\right)-(\sigma(\beta-\alpha)-1) t\right] \int_{\gamma}^{\delta}\left(\int_{0}^{s}(s-m)^{q-1} f(m) d m\right) d s \\
& -\frac{1}{\Delta \Gamma(q)}\left[\frac{\sigma}{2}\left(\beta^{2}-\alpha^{2}\right)-(\sigma(\beta-\alpha)-1) t\right] \int_{0}^{1}(1-s)^{q-1} f(s) d s,
\end{aligned}
$$

Observe that

$$
\begin{aligned}
& \left\|h_{n}(t)-h_{*}(t)\right\|=\| \frac{1}{\Gamma(q)} \int_{0}^{t}(t-s)^{q-1}\left(f_{n}(s)-f_{*}(s)\right) d s \\
& +\frac{\sigma}{\Delta \Gamma(q)}\left[-\left(\frac{\eta}{2}\left(\delta^{2}-\gamma^{2}\right)-1\right)+t(\eta(\delta-\gamma)-1)\right] \int_{\alpha}^{\beta}\left(\int_{0}^{s}(s-m)^{q-1}\left(f_{n}(m)-f_{*}(m)\right) d m\right) d s \\
& +\frac{\eta}{\Delta \Gamma(q)}\left[\frac{\sigma}{2}\left(\beta^{2}-\alpha^{2}\right)-(\sigma(\beta-\alpha)-1) t\right] \int_{\gamma}^{\delta}\left(\int_{0}^{s}(s-m)^{q-1}\left(f_{n}(m)-f_{*}(m)\right) d m\right) d s \\
& -\frac{1}{\Delta \Gamma(q)}\left[\frac{\sigma}{2}\left(\beta^{2}-\alpha^{2}\right)-(\sigma(\beta-\alpha)-1) t\right] \int_{0}^{1}(1-s)^{q-1}\left(f_{n}(s)-f_{*}(s)\right) d s \| \rightarrow 0,
\end{aligned}
$$

as $n \rightarrow \infty$. 
Thus, it follows by Lemma 4.6 that $\theta$ o $S_{F}$ is a closed graph operator. Further, we have $h_{n}(t) \in \Theta\left(S_{F, x_{n}}\right)$. Since $x_{n} \rightarrow x_{*}$, therefore, we have

$$
\begin{aligned}
h_{*}(t)= & \frac{1}{\Gamma(q)} \int_{0}^{t}(t-s)^{q-1} f_{*}(s) d s \\
& +\frac{\sigma}{\Delta \Gamma(q)}\left[-\left(\frac{\eta}{2}\left(\delta^{2}-\gamma^{2}\right)-1\right)+t(\eta(\delta-\gamma)-1)\right] \int_{\alpha}^{\beta}\left(\int_{0}^{s}(s-m)^{q-1} f_{*}(m) d m\right) d s \\
& +\frac{\eta}{\Delta \Gamma(q)}\left[\frac{\sigma}{2}\left(\beta^{2}-\alpha^{2}\right)-(\sigma(\beta-\alpha)-1) t\right] \int_{\gamma}^{\delta}\left(\int_{0}^{s}(s-m)^{q-1} f_{*}(m) d m\right) d s \\
& -\frac{1}{\Delta \Gamma(q)}\left[\frac{\sigma}{2}\left(\beta^{2}-\alpha^{2}\right)-(\sigma(\beta-\alpha)-1) t\right] \int_{0}^{1}(1-s)^{q-1} f_{*}(s) d s
\end{aligned}
$$

for some $f_{*} \in S_{F, x_{*}}$.

Finally, we show there exists an open set $U \subseteq C([0,1], \mathbb{R})$ with $x \notin \Omega_{F}(x)$ for any $\lambda \in$ $(0,1)$ and all $x \in \partial U$. Let $\lambda \in(0,1)$ and $x \in \lambda \Omega_{F}(x)$. Then there exists $f \in L^{1}([0,1], \mathbb{R})$ with $f \in S_{F, x}$ such that, for $t \in[0,1]$, we have

$$
\begin{aligned}
& h(t)=\frac{1}{\Gamma(q)} \int_{0}^{t}(t-s)^{q-1} f(s) d s \\
& +\frac{\sigma}{\Delta \Gamma(q)}\left[-\left(\frac{\eta}{2}\left(\delta^{2}-\gamma^{2}\right)-1\right)+t(\eta(\delta-\gamma)-1)\right] \int_{\alpha}^{\beta}\left(\int_{0}^{s}(s-m)^{q-1} f(m) d m\right) d s \\
& +\frac{\eta}{\Delta \Gamma(q)}\left[\frac{\sigma}{2}\left(\beta^{2}-\alpha^{2}\right)-(\sigma(\beta-\alpha)-1) t\right] \int_{\gamma}^{\delta}\left(\int_{0}^{s}(s-m)^{q-1} f(m) d m\right) d s \\
& -\frac{1}{\Delta \Gamma(q)}\left[\frac{\sigma}{2}\left(\beta^{2}-\alpha^{2}\right)-(\sigma(\beta-\alpha)-1) t\right] \int_{0}^{1}(1-s)^{q-1} f(s) d s
\end{aligned}
$$

and using the computations of the second step above we have.

$$
\begin{aligned}
|h(t)| \leq & \frac{\psi(\|x\|)}{\Gamma(q)}\left\{\left(1+\frac{\Delta_{1}}{|\Delta|}\right) \int_{0}^{1} p(s) d s+\frac{|\sigma| \Delta_{2}}{|\Delta|} \int_{\alpha}^{\beta}\left(\int_{0}^{s}(s-m)^{q-1} p(m) d m\right) d s\right. \\
& \left.+\frac{|\eta| \Delta_{1}}{|\Delta|} \int_{\gamma}^{\delta}\left(\int_{0}^{s}(s-m)^{q-1} p(m) d m\right) d s\right\} .
\end{aligned}
$$

Consequently, we have

$$
\begin{aligned}
& \|x\| \cdot\left\{\frac { \psi ( \| x \| ) } { \Gamma ( q ) } \left\{\left(1+\frac{\Delta_{1}}{|\Delta|}\right) \int_{0}^{1} p(s) d s+\frac{|\sigma| \Delta_{2}}{|\Delta|} \int_{\alpha}^{\beta}\left(\int_{0}^{s}(s-m)^{q-1} p(m) d m\right) d s\right.\right. \\
& \left.\left.+\frac{|\eta| \Delta_{1}}{|\Delta|} \int_{\gamma}^{\delta}\left(\int_{0}^{s}(s-m)^{q-1} p(m) d m\right) d s\right\}\right\}^{-1} \leq 1 .
\end{aligned}
$$

In view of $\left(A_{10}\right)$, there exists $M$ such that $\|x\| \neq M$. Let us set

$$
U=\{x \in C([0,1], \mathbb{R}):\|x\|<M\} .
$$


Note that the operator $\Omega_{F}: \bar{U} \rightarrow \mathcal{P}(C([0,1], \mathbb{R}))$ is upper semicontinuous and completely continuous. From the choice of $U$, there is no $x \in \partial U$ such that $x \in \lambda \Omega_{F}$ $(x)$ for some $\lambda \in(0,1)$. Consequently, by the nonlinear alternative of Leray-Schauder type (Lemma 4.5), we deduce that $\Omega_{F}$ has a fixed point $x \in \bar{U}$ which is a solution of the problem (1.1). This completes the proof. $\square$

Example 4.11 Consider the following strip fractional boundary value problem

$$
\left\{\begin{array}{l}
{ }^{c} D^{3 / 2} x(t) \in F(t, x(t)), 0<t<1, \\
x(0)=\int_{1 / 3}^{1 / 2} x(s) d s, x(1)=\int_{2 / 3}^{3 / 4} x(s) d s .
\end{array}\right.
$$

Here, $q=3 / 2, \sigma=1, \eta=1, \alpha=1 / 3, \beta=1 / 2, \gamma=2 / 3, \delta=3 / 4$, and $F:[0,1] \times \mathbb{R} \rightarrow \mathcal{P}(\mathbb{R})$ is a multivalued map given by

$$
x \rightarrow F(t, x) \in\left[\frac{|x|^{3}}{|x|^{3}+3}+3 t^{3}+5, \frac{|x|}{|x|+1}+t+1\right] .
$$

For $f \in F$, we have

$$
|f| \leq \max \left(\frac{|x|^{3}}{|x|^{3}+3}+3 t^{3}+5, \frac{|x|}{|x|+1}+t+1\right) \leq 9, \quad x \in \mathbb{R} .
$$

Thus,

$$
\|F(t, x)\|_{\mathcal{P}}:=\sup \{|y|: y \in F(t, x)\} \leq 9=p(t) \psi(\|x\|), x \in \mathbb{R},
$$

with $p(t)=1, \psi(\|x\|)=9$.

Further, we see that $\left(H_{3}\right)$ is satisfied with $M>20.679031$. Clearly, all the conditions of Theorem 4.10 are satisfied. So there exists at least one solution of the problem (4.2) on $[0,1]$.

\subsection{The lower semicontinuous case}

As a next result, we study the case when $F$ is not necessarily convex valued. Our strategy to deal with this problem is based on the nonlinear alternative of Leray Schauder type together with the selection theorem of Bressan and Colombo [35] for lower semicontinuous maps with decomposable values.

Theorem 4.12 Assume that $\left(A_{10}\right),\left(H_{2}\right)$ and the following condition holds:

$\left(H_{4}\right) F:[0,1] \times \mathbb{R} \rightarrow \mathcal{P}(\mathbb{R})$ is a nonempty compact-valued multivalued map such that

(a) $(t, x) \mapsto F(t, x)$ is $\Lambda \mathcal{L} \otimes \mathcal{B}$ measurable,

(b) $x \mapsto F(t, x)$ is lower semicontinuous for each $t \in[0,1]$;

Then the boundary value problem (1.2) has at least one solution on $[0,1]$.

Proof. It follows from $\left(\mathrm{H}_{2}\right)$ and $\left(\mathrm{H}_{4}\right)$ that $F$ is of l.s.c. type. Then from Lemma 4.7, there exists a continuous function $f: C([0,1], \mathbb{R}) \rightarrow L^{1}([0,1], \mathbb{R})$ such that $f(x) \in \mathcal{F}(x)$ for all $x \in C([0,1], \mathbb{R})$. 
Consider the problem

$$
\left\{\begin{array}{l}
{ }^{c} D^{q} x(t)=f(x(t)), \quad t \in[0,1] \\
x(0)=\sigma \int_{\alpha}^{\beta} x(s) d s, \quad x(1)=\eta \int_{\gamma}^{\delta} x(s) d s, \quad 0<\alpha<\beta<\gamma<\delta<1 .
\end{array}\right.
$$

Observe that if $x \in C^{2}([0,1], \mathbb{R})$ is a solution of (4.3), then $x$ is a solution to the problem (1.2). In order to transform the problem (4.3) into a fixed point problem, we define the operator $\overline{\Omega_{F}}$ as

$$
\begin{aligned}
\overline{\Omega_{F}} x(t)= & \frac{1}{\Gamma(q)} \int_{0}^{t}(t-s)^{q-1} f(x(s)) d s \\
& +\frac{\sigma}{\Delta \Gamma(q)}\left[-\left(\frac{\eta}{2}\left(\delta^{2}-\gamma^{2}\right)-1\right)+t(\eta(\delta-\gamma)-1)\right] \int_{\alpha}^{\beta}\left(\int_{0}^{s}(s-m)^{q-1} f(x(m)) d m\right) d s \\
& +\frac{\eta}{\Delta \Gamma(q)}\left[\frac{\sigma}{2}\left(\beta^{2}-\alpha^{2}\right)-(\sigma(\beta-\alpha)-1) t\right] \int_{\gamma}^{\delta}\left(\int_{0}^{s}(s-m)^{q-1} f(x(m)) d m\right) d s \\
& -\frac{1}{\Delta \Gamma(q)}\left[\frac{\sigma}{2}\left(\beta^{2}-\alpha^{2}\right)-(\sigma(\beta-\alpha)-1) t\right] \int_{0}^{1}(1-s)^{q-1} f(x(s)) d s .
\end{aligned}
$$

It can easily be shown that $\overline{\Omega_{F}}$ is continuous and completely continuous. The remaining part of the proof is similar to that of Theorem 3.1. So we omit it. This completes the proof. $\square$

\subsection{The Lipschitz case}

Now we prove the existence of solutions for the problem (1.2) with a nonconvex valued right-hand side by applying a fixed point theorem for multivalued map due to Covitz and Nadler [34].

Theorem 4.13 Assume that the following conditions hold:

$\left(H_{5}\right) F:[0,1] \times \mathbb{R} \rightarrow P_{c p}(\mathbb{R})$ is such that $F(\cdot, x):[0,1] \rightarrow P_{c p}(\mathbb{R})$ is measurable for each $x \in \mathbb{R}$.

$\left(H_{6}\right) \quad H_{d}(F(t, x), F(t, \bar{x})) \leq m(t)|x-\bar{x}|$ for almost all $t \in[0,1]$ and $x$, $\bar{x} \in \mathbb{R}$ with $m \in L^{1}\left([0,1], \mathbb{R}^{+}\right)$and $d(0, F(t, 0)) \leq m(t)$ for almost all $t \in[0,1]$.

Then the boundary value problem (1.2) has at least one solution on $[0,1]$ if

$$
\begin{aligned}
& \frac{1}{\Gamma(q)}\left\{\left(1+\frac{\Delta_{1}}{|\Delta|}\right) \int_{0}^{1}(1-s)^{q-1} m(s) d s+\frac{|\sigma| \Delta_{2}}{|\Delta|} \int_{\alpha}^{\beta}\left(\int_{0}^{s}(s-r)^{q-1} m(r) d r\right) d s\right. \\
& \left.+\frac{|\eta| \Delta_{1}}{|\Delta|} \int_{\gamma}^{\delta}\left(\int_{0}^{s}(s-r)^{q-1} m(r) d r\right) d s\right\}<1 .
\end{aligned}
$$

Proof. Observe that the set $S_{F, x}$ is nonempty for each $x \in C([0,1], \mathbb{R})$ by the assumption $\left(\mathrm{H}_{5}\right)$, so $F$ has a measurable selection (see Theorem III.6 [36]). Now we show that the operator $\Omega_{F}$, defined in the beginning of proof of Theorem 4.10, satisfies the assumptions of Lemma 4.8. To show that $\left.\Omega_{F}(x) \in P_{c l}(C[0,1], \mathbb{R})\right)$ for each $x \in C$ $([0,1], \mathbb{R})$, let $\left\{u_{n}\right\}_{n \geq 0} \in \Omega_{F}(x)$ be such that $u_{n} \rightarrow u(n \rightarrow \infty)$ in $C([0,1], \mathbb{R})$. Then $u$ $\in C([0,1], \mathbb{R})$ and there exists $v_{n} \in S_{F, x_{n}}$ such that, for each $t \in[0,1]$, 


$$
\begin{aligned}
u_{n}(t)= & \frac{1}{\Gamma(q)} \int_{0}^{t}(t-s)^{q-1} v_{n}(s) d s \\
& +\frac{\sigma}{\Delta \Gamma(q)}\left[-\left(\frac{\eta}{2}\left(\delta^{2}-\gamma^{2}\right)-1\right)+t(\eta(\delta-\gamma)-1)\right] \int_{\alpha}^{\beta}\left(\int_{0}^{s}(s-m)^{q-1} v_{n}(m) d m\right) d s \\
& +\frac{\eta}{\Delta \Gamma(q)}\left[\frac{\sigma}{2}\left(\beta^{2}-\alpha^{2}\right)-(\sigma(\beta-\alpha)-1) t\right] \int_{\gamma}^{\delta}\left(\int_{0}^{s}(s-m)^{q-1} v_{n}(m) d m\right) d s \\
& -\frac{1}{\Delta \Gamma(q)}\left[\frac{\sigma}{2}\left(\beta^{2}-\alpha^{2}\right)-(\sigma(\beta-\alpha)-1) t\right] \int_{0}^{1}(1-s)^{q-1} v_{n}(s) d s .
\end{aligned}
$$

As $F$ has compact values, we pass onto a subsequence to obtain that $v_{n}$ converges to $v$ in $L^{1}([0,1], \mathbb{R})$. Thus, $v \in S_{F, x}$ and for each $t \in[0,1]$,

$$
\begin{aligned}
u_{n}(t) \rightarrow u(t)= & \frac{1}{\Gamma(q)} \int_{0}^{t}(t-s)^{q-1} v(s) d s \\
& +\frac{\sigma}{\Delta \Gamma(q)}\left[-\left(\frac{\eta}{2}\left(\delta^{2}-\gamma^{2}\right)-1\right)+t(\eta(\delta-\gamma)-1)\right] \int_{\alpha}^{\beta}\left(\int_{0}^{s}(s-m)^{q-1} v_{n}(m) d m\right) d s \\
& +\frac{\eta}{\Delta \Gamma(q)}\left[\frac{\sigma}{2}\left(\beta^{2}-\alpha^{2}\right)-(\sigma(\beta-\alpha)-1) t\right] \int_{\gamma}^{\delta}\left(\int_{0}^{s}(s-m)^{q-1} v_{n}(m) d m\right) d s \\
& -\frac{1}{\Delta \Gamma(q)}\left[\frac{\sigma}{2}\left(\beta^{2}-\alpha^{2}\right)-(\sigma(\beta-\alpha)-1) t\right] \int_{0}^{1}(1-s)^{q-1} v_{n}(s) d s .
\end{aligned}
$$

Hence, $u \in \Omega(x)$.

Next, we show that there exists $\gamma<1$ such that

$$
H_{d}\left(\Omega_{F}(x), \Omega_{F}(\bar{x})\right) \leq \gamma\|x-\bar{x}\| \quad \text { for each } \quad x, \bar{x} \in C([0,1], \mathbb{R}) .
$$

Let $x, \bar{x} \in C([0,1], \mathbb{R})$ and $h_{1} \in \square(x)$. Then there exists $v_{1}(t) \in F(t, x(t))$ such that, for each $t \in[0,1]$,

$$
\begin{aligned}
h_{1}(t)= & \frac{1}{\Gamma(q)} \int_{0}^{t}(t-s)^{q-1} v_{1}(s) d s \\
& +\frac{\sigma}{\Delta \Gamma(q)}\left[-\left(\frac{\eta}{2}\left(\delta^{2}-\gamma^{2}\right)-1\right)+t(\eta(\delta-\gamma)-1)\right] \int_{\alpha}^{\beta}\left(\int_{0}^{s}(s-m)^{q-1} v_{1}(m) d m\right) d s \\
& +\frac{\eta}{\Delta \Gamma(q)}\left[\frac{\sigma}{2}\left(\beta^{2}-\alpha^{2}\right)-(\sigma(\beta-\alpha)-1) t\right] \int_{\gamma}^{\delta}\left(\int_{0}^{s}(s-m)^{q-1} v_{1}(m) d m\right) d s \\
& -\frac{1}{\Delta \Gamma(q)}\left[\frac{\sigma}{2}\left(\beta^{2}-\alpha^{2}\right)-(\sigma(\beta-\alpha)-1) t\right] \int_{0}^{1}(1-s)^{q-1} v_{1}(s) d s .
\end{aligned}
$$

By $\left(H_{7}\right)$, we have

$$
H_{d}(F(t, x), F(t, \bar{x})) \leq m(t)|x(t)-\bar{x}(t)| .
$$

So, there exists $w \in F(t, \bar{x}(t))$ such that

$$
\left|v_{1}(t)-w\right| \leq m(t)|x(t)-\bar{x}(t)|, \quad t \in[0,1] .
$$

Define $U:[0,1] \rightarrow \mathcal{P}(\mathbb{R})$ by

$$
U(t)=\left\{w \in \mathbb{R}:\left|v_{1}(t)-w\right| \leq m(t)|x(t)-\bar{x}(t)|\right\} .
$$


Since the multivalued operator $U(t) \cap F(t, \bar{x}(t))$ is measurable [36, Proposition III.4], there exists a function $v_{2}(t)$ which is a measurable selection for $U$. So $v_{2}(t) \in F(t, \bar{x}(t))$ and for each $t \in\left[\begin{array}{ll}0, & 1\end{array}\right]$, we have $\left|v_{1}(t)-v_{2}(t)\right| \leq m(t)|x(t)-\bar{x}(t)|$.

For each $t \in[0,1]$, let us define

$$
\begin{aligned}
& h_{2}(t)=\frac{1}{\Gamma(q)} \int_{0}^{t}(t-s)^{q-1} v_{2}(s) d s \\
& +\frac{\sigma}{\Delta \Gamma(q)}\left[-\left(\frac{\eta}{2}\left(\delta^{2}-\gamma^{2}\right)-1\right)+t(\eta(\delta-\gamma)-1)\right] \int_{\alpha}^{\beta}\left(\int_{0}^{s}(s-m)^{q-1} v_{2}(m) d m\right) d s \\
& +\frac{\eta}{\Delta \Gamma(q)}\left[\frac{\sigma}{2}\left(\beta^{2}-\alpha^{2}\right)-(\sigma(\beta-\alpha)-1) t\right] \int_{\gamma}^{\delta}\left(\int_{0}^{s}(s-m)^{q-1} v_{2}(m) d m\right) d s \\
& -\frac{1}{\Delta \Gamma(q)}\left[\frac{\sigma}{2}\left(\beta^{2}-\alpha^{2}\right)-(\sigma(\beta-\alpha)-1) t\right] \int_{0}^{1}(1-s)^{q-1} v_{2}(s) d s .
\end{aligned}
$$

Thus,

$$
\begin{aligned}
& \left|h_{1}(t)-h_{2}(t)\right|=\frac{1}{\Gamma(q)} \int_{0}^{t}(t-s)^{q-1}\left|v_{1}(s)-v_{2}(s)\right| d s \\
& +\left|\frac{\sigma}{\Delta \Gamma(q)}\left[-\left(\frac{\eta}{2}\left(\delta^{2}-\gamma^{2}\right)-1\right)+t(\eta(\delta-\gamma)-1)\right]\right| \int_{\alpha}^{\beta}\left(\int_{0}^{s}(s-r)^{q-1}\left|v_{1}(r)-v_{2}(r)\right| d r\right) d s \\
& \quad+\left|\frac{\eta}{\Delta \Gamma(q)}\left[\frac{\sigma}{2}\left(\beta^{2}-\alpha^{2}\right)-(\sigma(\beta-\alpha)-1) t\right]\right| \int_{\gamma}^{\delta}\left(\int_{0}^{s}(s-r)^{q-1}\left|v_{1}(r)-v_{2}(r)\right| d r\right) d s \\
& \quad+\left|\frac{1}{\Delta \Gamma(q)}\left[\frac{\sigma}{2}\left(\beta^{2}-\alpha^{2}\right)-(\sigma(\beta-\alpha)-1) t\right]\right| \int_{0}^{1}(1-s)^{q-1}\left|v_{1}(s)-v_{2}(s)\right| d s \\
& \leq \frac{\|x-\bar{x}\|}{\Gamma(q)}\left\{\left(1+\frac{\Delta_{1}}{|\Delta|}\right) \int_{0}^{1}(1-s)^{q-1} m(s) d s+\frac{|\sigma| \Delta_{2}}{|\Delta|} \int_{\alpha}^{\beta}\left(\int_{0}^{s}(s-r)^{q-1} m(r) d r\right) d s\right. \\
& \left.\quad+\frac{|\eta| \Delta_{1}}{|\Delta|} \int_{\gamma}^{\delta}\left(\int_{0}^{s}(s-r)^{q-1} m(r) d r\right) d s\right\} .
\end{aligned}
$$

Hence,

$$
\begin{aligned}
\left\|h_{1}-h_{2}\right\| \leq & \frac{1}{\Gamma(q)}\left\{\left(1+\frac{\Delta_{1}}{|\Delta|}\right) \int_{0}^{1}(1-s)^{q-1} m(s) d s+\frac{|\sigma| \Delta_{2}}{|\Delta|} \int_{\alpha}^{\beta}\left(\int_{0}^{s}(s-r)^{q-1} m(r) d r\right) d s\right. \\
& \left.+\frac{|\eta| \Delta_{1}}{|\Delta|} \int_{\gamma}^{\delta}\left(\int_{0}^{s}(s-r)^{q-1} m(r) d r\right) d s\right\}\|x-\bar{x}\| .
\end{aligned}
$$

Analogously, interchanging the roles of $x$ and $\bar{x}$, we obtain

$$
\begin{aligned}
& H_{d}\left(\Omega_{F}(x), \Omega_{F}(\bar{x})\right) \\
\leq & \gamma\|x-\bar{x}\| \\
\leq & \frac{1}{\Gamma(q)}\left\{\left(1+\frac{\Delta_{1}}{|\Delta|}\right) \int_{0}^{1}(1-s)^{q-1} m(s) d s \frac{|\sigma| \Delta_{2}}{|\Delta|} \int_{\alpha}^{\beta}\left(\int_{0}^{s}(s-r)^{q-1} m(r) d r\right) d s\right. \\
& \left.+\frac{|\eta| \Delta_{1}}{|\Delta|} \int_{\gamma}^{\delta}\left(\int_{0}^{s}(s-r)^{q-1} m(r) d r\right) d s\right\}\|x-\bar{x}\| .
\end{aligned}
$$




\author{
Since $\Omega_{F}$ is a contraction, it follows by Lemma 4.8 that $\square$ has a fixed point $x$ which is \\ a solution of (1.2). This completes the proof. $\square$
}

\title{
Acknowledgements
}

The authors were grateful to the reviewers for their useful comments. The research of B. Ahmad was supported by Deanship of Scientific Research (DSR), King Abdulaziz University, Jeddah, Saudi Arabia.

\section{Author details}

'Department of Mathematics, Faculty of Science, King Abdulaziz University, P.O. Box 80203, Jeddah 21589, Saudi Arabia ${ }^{2}$ Department of Mathematics, University of loannina 45110 loannina, Greece

\section{Authors' contributions}

Each of the authors, BA and SKN contributed to each part of this study equally and read and approved the final version of the manuscript.

\section{Competing interests}

The authors declare that they have no competing interests.

Received: 30 December 2011 Accepted: 9 May 2012 Published: 9 May 2012

\section{References}

1. Agarwal, RP, Belmekki, M, Benchohra, M: A survey on semilinear differential equations and inclusions involving Riemann-Liouville fractional derivative. Adv. Diff. Equ 2009(47) (2009). Art. ID 981728

2. Ahmad, B, Nieto, JJ: Existence of solutions for nonlocal boundary value problems of higher order nonlinear fractional differential equations. Abstr. Appl. Anal 2009(9) (2009). Article ID 494720

3. Ahmad, B, Nieto, JJ: Existence results for nonlinear boundary value problems of fractional integrodifferential equations with integral boundary conditions. Bound. Value Probl 2009(11) (2009). Art. ID 708576

4. Ahmad, B, Nieto, JJ: Existence results for a coupled system of nonlinear fractional differential equations with three-point boundary conditions. Comput. Math. Appl. 58, 1838-1843 (2009). doi:10.1016/j.camwa.2009.07.091

5. Agarwal, RP, Andrade, B, Cuevas, C: Weighted pseudo-almost periodic solutions of a class of semilinear fractional differential equations. Nonlinear Anal. Real World Appl. 11, 3532-3554 (2010). doi:10.1016/j.nonrwa.2010.01.002

6. Ahmad, B, Ntouyas, SK: Some existence results for boundary value problems for fractional differential inclusions with non-separated boundary conditions. Electron. J. Qual. Theory Diff. Equ. 2010(17):71 (2010)

7. Ahmad, B, Sivasundaram, S: On four-point nonlocal boundary value problems of nonlinear integro-differential equations of fractional order. Appl. Math. Comput. 217, 480-487 (2010). doi:10.1016/j.amc.2010.05.080

8. Ahmad, B: Existence of solutions for irregular boundary value problems of non-linear fractional differential equations. Appl. Math. Lett. 23, 390-394 (2010). doi:10.1016/j.aml.2009.11.004

9. Bai, ZB: On positive solutions of a nonlocal fractional boundary value problem. Nonlinear Anal. 72, 916-924 (2010). doi:10.1016/j.na.2009.07.033

10. Balachandran, K, Trujillo, JJ: The nonlocal Cauchy problem for nonlinear fractional integrodifferential equations in Banach spaces. Nonlinear Anal. 72, 4587-4593 (2010). doi:10.1016/j.na.2010.02.035

11. Zhang, S: Positive solutions to singular boundary value problem for nonlinear fractional differential equation. Comput. Math. Appl. 59, 1300-1309 (2010). doi:10.1016/j.camwa.2009.06.034

12. Baleanu, D, Mustafa, OG, Agarwal, RP: An existence result for a superlinear fractional differential equation. Appl. Math. Lett. 23, 1129-1132 (2010). doi:10.1016/j.aml.2010.04.049

13. Baleanu, D, Mustafa, OG: On the global existence of solutions to a class of fractional differential equations. Comput. Math. Appl. 59, 1835-1841 (2010). doi:10.1016/j.camwa.2009.08.028

14. Agarwal, RP, Ahmad, B: Existence theory for anti-periodic boundary value problems of fractional differential equations and inclusions. Comput. Math. Appl. 62, 1200-1214 (2011). doi:10.1016/j.camwa.2011.03.001

15. Ahmad, B, Ntouyas, SK: Existence results for nonlinear fractional differential equations with four-point nonlocal type integral boundary conditions. Afr. Diaspora J. Math. 11, 29-39 (2011)

16. Baleanu, D, Mustafa, OG, O'Regan, D: A Nagumo-like uniqueness theorem for fractional differential equations. J. Phys. A, Math. Theor 44(39) (2011). Article ID 392003, 6

17. Ahmad, B, Nieto, JJ: Riemann-Liouville fractional integro-differential equations with fractional nonlocal integral boundary conditions. Bound. Value Probl. 2011(9):36 (2011)

18. Ahmad, B, Nieto, JJ, Alsaedi, A, El-Shahed, M: A study of nonlinear Langevin equation involving two fractional orders in different intervals. Nonlinear Anal. Real World Appl. 13, 599-606 (2012). doi:10.1016/j.nonrwa.2011.07.052

19. Baleanu, D, Diethelm, K, Scalas, E, Trujillo, JJ: Fractional calculus models and numerical methods. Series on Complexity, Nonlinearity and Chaos. World Scientific, Boston (2012)

20. Ahmad, B, Alsaedi, A, Alghamdi, B: Analytic approximation of solutions of the forced Duffing equation with integral boundary conditions. Nonlinear Anal. Real World Appl. 9, 1727-1740 (2008). doi:10.1016/j.nonrwa.2007.05.005

21. Boucherif, A: Second-order boundary value problems with integral boundary conditions. Nonlinear Anal. 70, 364-371 (2009). doi:10.1016/j.na.2007.12.007

22. Ahmad, B, Hayat, T, Asghar, S: Diffraction of a plane wave by an elastic knife-edge adjacent to a strip. Canad. Appl. Math. Quart. 9, 303-316 (2001)

23. Asghar, S, Ahmad, B, Ayub, M: Diffraction from an absorbing half plane due to a finite cylindrical source. Acustica-Acta Acustica. 82, 365-367 (1996)

24. Kilbas, AA, Srivastava, HM, Trujillo, JJ: Theory and Applications of Fractional Differential Equations. North-Holland Mathematics Studies, 204. Elsevier Science B.V., Amsterdam (2006) 
25. Podlubny, I: Fractional Differential Equations. Academic Press, San Diego (1999)

26. Samko, SG, Kilbas, AA, Marichev, Ol: Fractional Integrals and Derivatives, Theory and Applications. Gordon and Breach, Yverdon (1993)

27. Krasnoselskii, MA: Two remarks on the method of successive approximations. Uspekhi Mat. Nauk. 10, 123-127 (1955)

28. Deimling, K: Multivalued Differential Equations. Walter De Gruyter, Berlin-New York (1992)

29. Hu, Sh, Papageorgiou, N: Handbook of Multivalued Analysis, Theory I. Kluwer, Dordrecht (1997)

30. Kisielewicz, M: Differential Inclusions and Optimal Control. Kluwer, Dordrecht, The Netherlands (1991)

31. Granas, A, Dugundji, J: Fixed Point Theory. Springer-Verlag, New York (2005)

32. Lasota, A, Opial, Z: An application of the Kakutani-Ky Fan theorem in the theory of ordinary differential equations. Bull. Acad. Polon. Sci. Ser. Sci. Math. Astronom. Phys. 13, 781-786 (1965)

33. Frigon, M: Théorèmes d'existence de solutions d'inclusions différentielles. In: Granas, A, Frigon, M (eds.) Topological Methods in Differential Equations and Inclusions, NATO ASI Series C, vol. 472, pp. 51-87. Kluwer Acad. Publ., Dordrecht (1995)

34. Covitz, H, Nadler, SB Jr: Multivalued contraction mappings in generalized metric spaces. Israel J. Math. 8, 5-11 (1970). doi:10.1007/BF02771543

35. Bressan, A, Colombo, G: Extensions and selections of maps with decomposable values. Studia Math. 90, 69-86 (1988)

36. Castaing, C, Valadier, M: Convex Analysis and Measurable Multifunctions, Lecture Notes in Mathematics 580. SpringerVerlag, New York (1977)

doi:10.1186/1687-2770-2012-55

Cite this article as: Ahmad and Ntouyas: Existence results for nonlocal boundary value problems of fractional differential equations and inclusions with strip conditions. Boundary Value Problems 2012 2012:55.

\section{Submit your manuscript to a SpringerOpen ${ }^{\odot}$} journal and benefit from:

- Convenient online submission

- Rigorous peer review

- Immediate publication on acceptance

- Open access: articles freely available online

- High visibility within the field

- Retaining the copyright to your article

Submit your next manuscript at $\gg$ springeropen.com 